\title{
ESSAI DE CULTURE IN VITRO
}

\section{DE MICROFILAIRES DE BANCROFT}

\section{Par F. COUTELEN}

Dans une note antérieure, parue dans le Bulletin de la Société de Pathologie exotique et intitulée «Contribution aux essais de culture in vitro d'embryons de filaires », nous avons indiqué les survies d'embryons d'Icosiella neglecta (Diesing, 1851) que nous avons obtenues en milieu hypotonique liquide de Ponselle à la température de $18^{\circ} \mathrm{C}$. Au cours de la revue bibliographique et critique qui précédait nos propres expériences, nous avons relaté, entre autres, les résultats obtenus par Takeshita et Okuda avec des embryons de Wuchereria bancrofti (Cobbold, 1877). En 1925, ces deux auteurs japonais, plaçant des embryons de cette filaire dans e deux appareils spécialement construits à cet usage », obtinrent des survies de 12 à 14 jours en solution physiologique de $\mathrm{NaCl}$ et de 23 jours, à $11^{\circ} 5 \mathrm{c}$, en « solution saline, hémoglobinisée avec du sang de bœuf » (?) ( cattle hæmoglobinized saline »). Nous mentionnions déjà que nous n'avions connaissance de ces résultats que par une analyse du Tropical Diseases Bulletin; tirée elle-même d'un sommaire paru dans le Japan Medical World. Depuis, il ne nous a pas encore été possible de retrouver le texte original, en sorte que nous ignorons toujours les détails techniques de cette expérience et, en particulier, l'agencement des « deux appareils spécialement construits à cet usage ».

Certains points importants de la biologie des microfilaires étant encore inconnus ou controversés, tels que la signification et la destinée du corps central, l'origine de la gaine, le mode de sortie de l'embryon de sa gaine et l'existence d'un dard céphalique, il nous a paru intéressant d'essayer, à notre tour, des cultures in vitro, afin de préciser, si possible, ces différentes particularités.

Ayant eu dernièrement l'occasion d'examiner un malade atteint de filariose nocturne, nous l'avons trouvé porteur d'un nombre suffisant de microfilaires dans son sang périphérique (25 embryons par goutte de sang sous lamelle $22 \times 22$ ) pour faire les essais suivants :

Technique. - Par ponction veineuse au pli du coude, nous avons fait une prise de sang aseptique de $50 \mathrm{~cm}^{\mathrm{a}}$ environ. Après coagula-

Annales de Parasitologie, T. VII, $\mathrm{N}^{\circ}$ 5. $-1^{\text {er }}$ septembre 1929 , p. 399-409. 
tion, nous avons recueilli le sérum du malade avec une pipette à houle et nous l'avons réparti en quantités égales dans trois tubes à essais.

Le premier tube à essai, contenant du sérum seul, nous a servi de témoin.

Le deuxième tube à essai a reçu un volume de solution physiologique de $\mathrm{NaCl}$ stérile égal au volume de sérum qu'il contenait.

Le troisième tube à essai a reçu tous les quatre jours deux centimètres cubes d'une solution stérile de glycose à 1 p. 100 dans la solution physiologique de $\mathrm{NaCl}$. Les milieux de ces deux derniers tubes ont été dédoublés et plus ou moins modifiés par la suite.

Ces tubes, bouchés à l'ouate et capuchonnés, ont été abandonnés à la température du laboratoire. Leur contenu a été régulièrement examiné, soit en prélevant directement au fond du tube une goutte du milieu, soit en examinant le culot de centrifugation d'une faible quantité de la culture (tube $\mathrm{n}^{\circ} 3$ ).

L'examen microscopique a été fait soit à frais, entre lame et lamelle, avec ou sans coloration vitale au rouge neutre, soit sur frottis avec coloration post-vitale au bleu de Sabrazès, soit sur frottis ou gouttes épaisses colorés par la méthode panoptique.

Résultats. - Durant ces expériences, nous avons examiné et mesuré très exactement un grand nombre d'embryons ; nous avons groupé dans le tableau $\mathrm{n}^{\circ} 1$ qui accompagne cette note, les mensurations se rapportant à une dizaine de microfilaires seulement, pour ne pas surcharger nos pages de chiffres, mais en ayant soin de choisir ces dix échantillons parmi ceux qui présentaient non seulement des chiffres moyens, mais aussi des chiffres extrêmes : nos moyennes sont done aussi exactes que possible.

I. - Voici d'abord quelques chiffres mesurant, en microns, les dimensions des microfilaires, le jour de la prise de sang (voir tableau $\mathrm{n}^{\circ}$ 1):

$1^{\circ}$ Gaines : Longueur minima : $360 \mu$; longueur maxima : $404 \mu$; longueur moyenne : $382 \mu$.

Largeur minima : 9,1 $\mu$; largeur maxima : 9,6 $\mu$; largeur moyenne : $9,3 \mu$.

Ces chiffres sont voisins de ceux donnés par Foley : $394 \mu$ et $8,25 \mu$.

$2^{\circ}$ Embryons : Longueur minima : $261 \mu$; longueur maxima : $306 \mu$; longueur moyenne : $283 \mu$.

Largeur minima: 5,5 $\mu$; largeur maxima : 5,5 $\mu$; largeur moyenne : $5,5 \mu$.

Foley donne les chiffres moyens de $275 \mu$ et $6,8 \mu$ assez voisins des nôtres ; par contre certains auteurs ont donné des chiffres moyens plus élevés : Manson, $333 \mu$ et de 7 à $8 \mu$; Penel, $300 \mu$ et $8 \mu$. Ces 
différences de taille s'expliquent probablement par l'espace de temps qui s'est écoulé entre la ponte des embryons et l'époque à laquelle on les a recherchés dans le sang périphérique et examinés.

Notons dès à présent qu'il n'y a aucune relation entre les dimensions de la gaine et celles de l'embryon correspondant. C'est ainsi, par exemple, qu'un petit embryon mesurant $261 \mu \times 5,5 \mu$ avait une gaine de $382 \mu \times 9,3 \mu$; qu'un gros embryon de $292 \mu \times 5,5 \mu$ avait Line pétite gaine de $360 \mu \times 9,1 \mu$ seulement.

Pour les distances qui séparent l'extrémité céphalique des embryons de leurs diverses " taches » nous avons trouvé des chiffres (voir tableau $\mathrm{n}^{\circ} 3$, microfilaire A) concordant à peu de chose près avec ceux donnés par Foley dans son excellent travail statistique.

II. - Voici maintenant les résultats généraux de nos essais de culture, in vitro, d'embryons de filaire de Bancroft:

A l'encontre des essais de Takeshita et Okuda, qui n'ont obtenu des survies de 12 à 23 jours qu'en milieux spéciaux et au moyen " d'appareils spécialement construits à cet usage », nous avons obtenu des cultures d'embryons de 32 jours sans aucun appareil, en tubes à essais ordinaires ; nous avons simplement employé les techniques bactériologiques habituelles que nous avions eu déjà l'occasion d'utiliser pour la culture de formes larvaires d'autres helminthes; de plus, les milieux constitués par la solution physiologique de $\mathrm{NaCl}$ simple ou hémoglobinisée ou dextrosée, ne nous ont pas donné, dans cette première série d'expériences du moins, de résultats meilleurs que le sérum humain normal, non chauffé et en qualité suffisante : c'est même dans le tube témoin que nous avons enregistré les survies les plus nómbreuses, les plus longues et les débuts d'évolution les plus marqués. Aussi, comme type d'évolution, nous allons passer en revue les modifications subies par les embryons conservés dans du sérum seul, en indiquant, chemin faisant, les changements de taille, toujours plus faibles, qu'ont présenté parallèlement les microfilaires des autres milieux (consulter la dernière colonne du tableau comparatif $n^{\circ} 1$ ).

Dans les tout premiers jours, le changement le plus important, observé chez les embryons, est la perte de leur gaine. Nous préciserons dans une note ultérieure la façon dont s'opère ce dégainement. Ce phénomène, général pour les microfilaires cultivées en sérum seul, s'observe exceptionnellement dans les milieux fluidifiés par addition de solutions physiologique de $\mathrm{NaCl}$ ou de glycose ; il paraît donc lié à la viscosité du milieu plutôt qu'à un abaissement de la température. Nous reviendrons ultérieurement sur ce point. A ce stade, la taille des embryons ne s'est

Annales de Parasitologie, t. VII, Na 5. - $1^{\text {er }}$ septembre 1929.

26. 
TABlead COMParatif $\mathrm{N}^{\circ} 1$

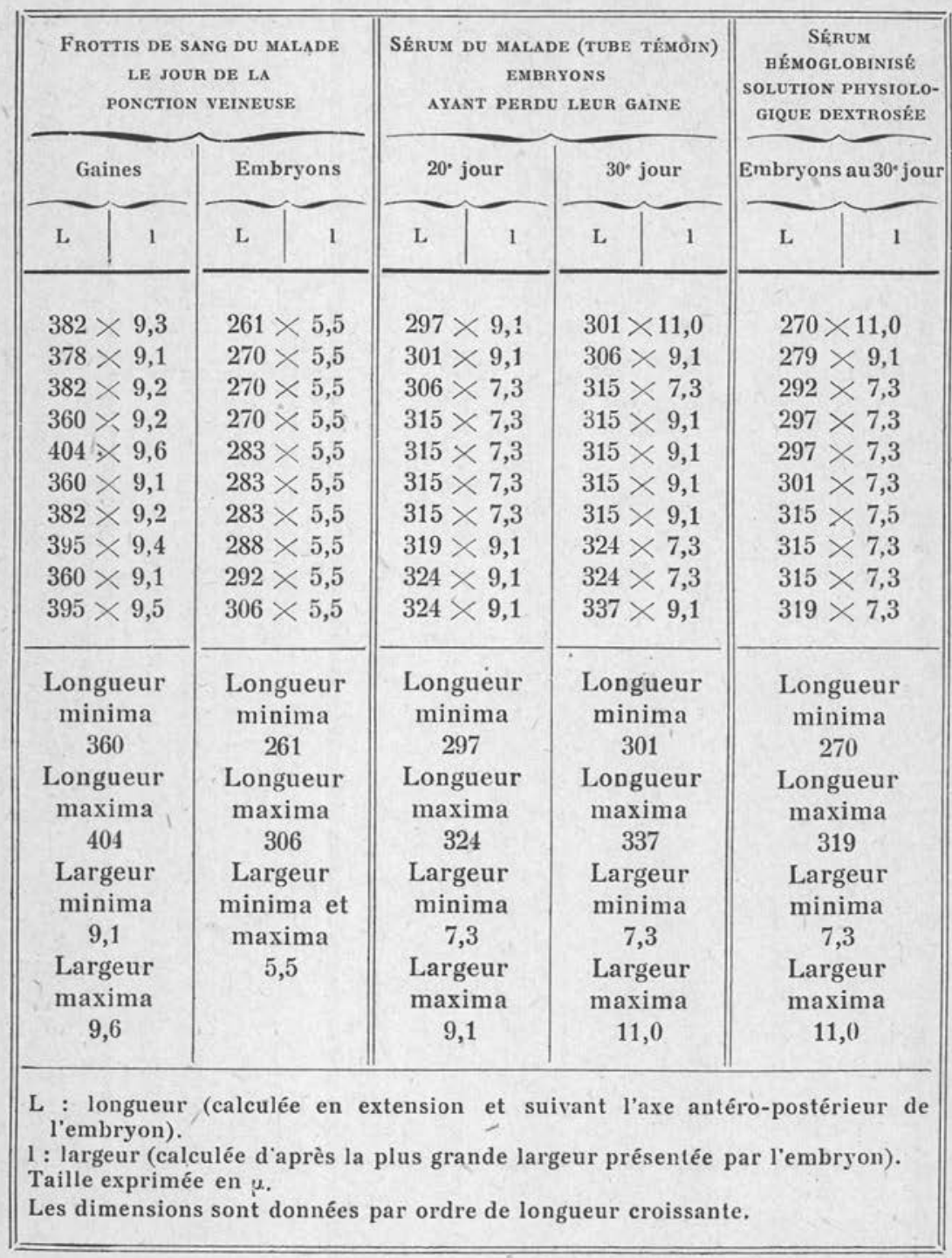

pas accrue, leur morphologie interne n'a pas changé : on distingue seulement, sur frottis colorés par la méthode panoptique, la tache oblique, la tache en $\mathrm{V}$, le corps central et la tache caudale ; nous avons très rarement observé la tache inconstante de Brumpt. Nos mesures étant exprimées en microns, voici quelques distances 
moyennes enregistrées : espace céphalique, $6 \mu$; espace caudal (sans cellules somatiques), $19 \mu$; distance de l'extrémité céphalique à la tache oblique, $58 \mu$; à la tache en $\mathrm{V}$, $90 \mu$; au milieu du corps central, $170 \mu$; à la tache caudale, $237 \mu$; ̀̀ l'extrémité caudale, $283 \mu$. Largeur moyenne des embryons, 5,5 $\mu$. Le nombre total moyen des cellules somatiques s'élève à 312 (Korke donne le chiffre de 313 ); elles sont réparties de la façon suivante : de l'extrémité céphalique à la tache en $\mathrm{V}, 120$; de la tache en V à la tache caudale, 164 ; de la tache caudale à l'extrémité caudale, 28. Nous avons volontairement laissé de côté le corps central, comme point de repère, car il est destiné à disparaître progressivement au cours de l'évolution.

En effet, dans les jours qui suivent, les embryons du milieu constitué par du sérum seul, destinés à survivre et à continuer leur évolution, après s'être débarrassés de leur gaine, perdent progressivement leur corps central. Cette formation, sur l'origine et la signification de laquelle nous reviendrons ultérieurement, perd ses contours nets, se résoud, petit à petit, en fines granulations qui, quelquefois, s'étalent, sans ordre apparent, sur toute la largeur de l'embryon. On

Fig. 1. - A. Microfilaire avec sa gaine, le jour de la prise de sang. B. Microfilaire au vingtième jour de culture en sérum non chauffé. C. Microfilaire au trentième jour de culture en sérum non chauffé. La microfilaire A présentait une largeur de $7 \mu$ dépassant de $1,5 \mu$ la largeur des autres microfilaires examinées; elle désavantage donc l'étude comparative de l'accroissement de taille des microfilaires $\mathrm{B}$ et $\mathrm{C}$; nous l'avons cependant fait figurer à cause de sa fixation et de sa coloration parfaites.
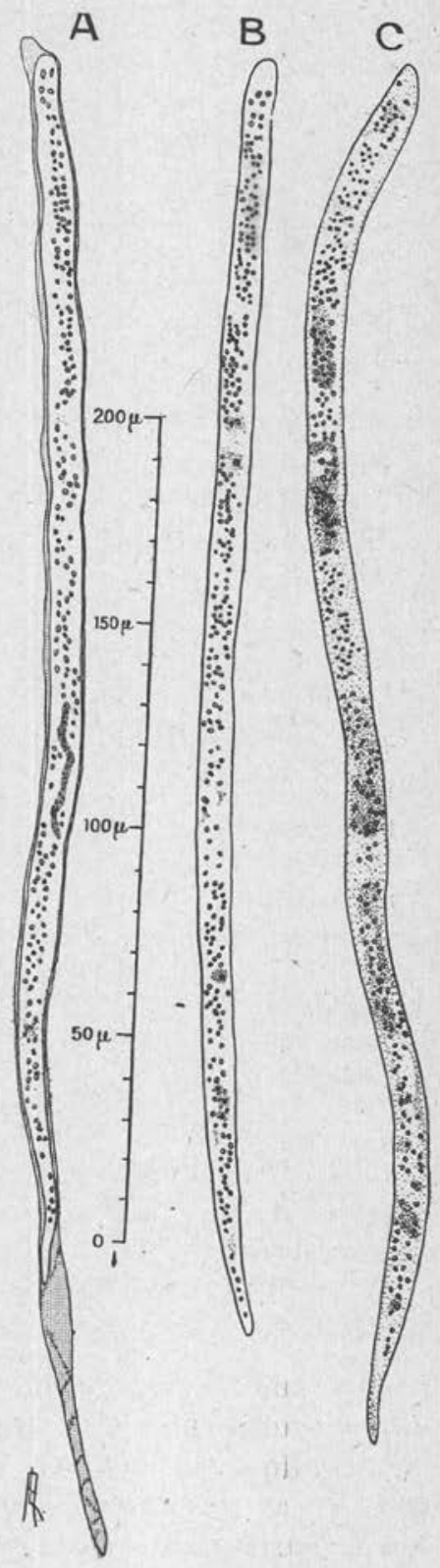
assiste progressivement à une véritable fonte du corps central. Bien loin de s'organiser, comme on l'a cru jusqu'ici, il se désagrège et finit par disparaître complètement.

La microfilaire B de nos figures 1 et 2 , au vingtième jour de culture, nous montre la disparition complète du corps central dont le seul vestige réside en un tout petit amas de quelques fines granulations.

A ce stade $\left(20^{\circ}\right.$ jour $)$, les embryons ont déjà grossi. Leur longueur moyenne a crû de $283 \mu$ à $310 \mu$; leur largeur moyenne de $5,5 \mu$ à $8,2 \mu$; la tache en V a été remplacée par l'ébauche du pore excréteur et la première cellule excrétrice; la $1^{\text {ro }}$ cellule génitale est apparue ; la tache caudale qui était à peine large de $5 \mu$, a été remplacée par les $2^{\circ}, 3^{\circ}$ et $4^{\circ}$ cellules génitales (1), ainsi que par l'ébauche du pore anal et leur ensemble a acquis une extension égale à $15 \mu$; sur cet échantillon est apparue nettement la tache inconstante de l'extrémité caudale. A remarquer, ici aussi, le déplacement vers l'arrière des dernières cellules somatiques, en sorte que l'espace caudal se trouve réduit de $19 \mu$ à $4 \mu$. En même temps que l'embryon croissait en longueur et en largeur, s'opérait une multiplication des noyaux somatiques surtout dans la région des ébauches exc̀rétrice et génitale : en effet, de l'extrémité céphalique au pore excréteur, le nombre des noyaux passait de 120 à 156 ; du pore excréteur au pore anal il passait de 164 à 181 , tandis que le nombre de noyaux répartis entre le pore anal et l'extrémité caudale restait égal à 28 .

Donc, en 20 jours de culture en sérum frais, les microfilaires avaient subi de notables changements morphologiques externes (disparition de la gaine, accroissement de la taille) et internes (disparition progressive du corps central interne, apparition du pore excréteur, de la première cellule excrétrice, des cellules génitales 1 , 2,3 et 4 , de l'ébauche dú pore anal, de la tache inconstante et multiplication des cellules somatiques). Dans les autres milieux les changements étaient moins accusés et la mortalité plus élevée. Il faut noter cependant que, les changements concernant la gaine et le corps central mis à part, ce stade d'évolution peut également s'observer sur des microfilaires dans le sang des malades.

Du vingtième au trentième jour, l'évolution s'était encore accentuée chez les exemplaires, encore nombreux, restés vivants ; à partir

(1) Nous continuons à adopter provisoirement l'appellation de a cellules génitales " donnée classiquement à ces cellules numérotées " 2,3 et 4 » tout en faisant des réserves sur leur véritable signification embryogénique non démontrée jusqu'à ce jour. La même réserve s'imposera lorsque, au cours de ce travail, nous appellerons nous-même "ébauches génitales 2,3 et 4 " les groupements cellulaires issus de ces " cellules génitales 2,3 et 4 ». 


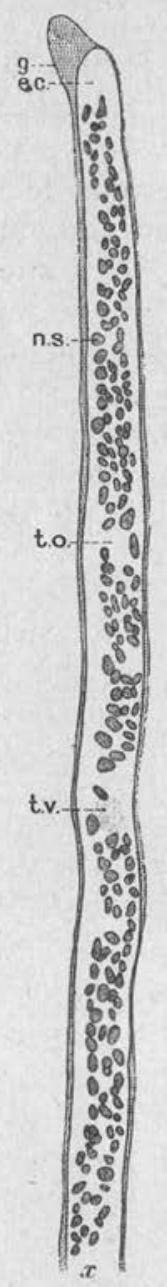

A
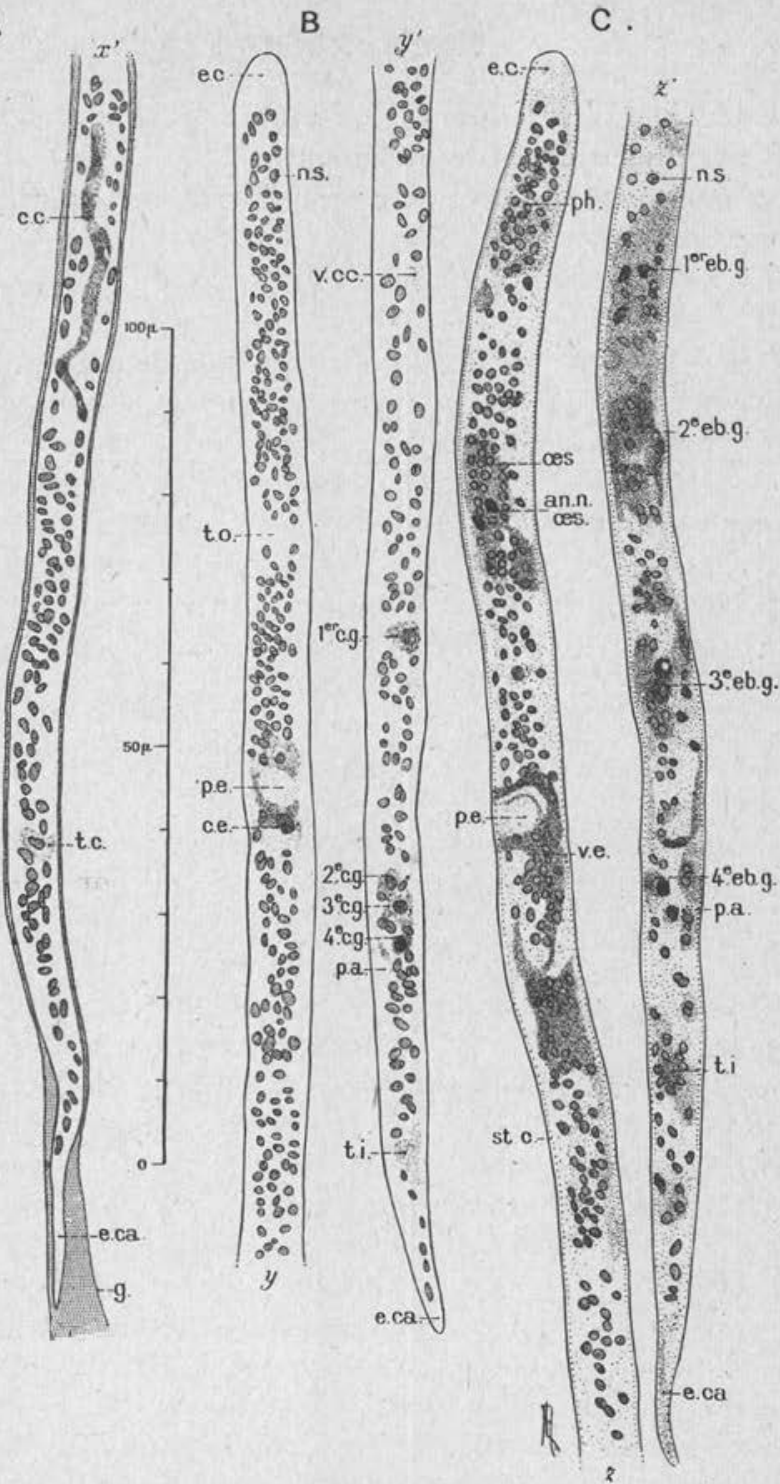

Fig. 2. - A, B et $\mathrm{C}$, les mêmes microfilaires dessinées à un fort grossissement. $g$, gaine; $e c$, espace céphalique; $n s$, noyaux des cellules somatiques; to, tache oblique ; $t v$, tache en V; $c c$, corps central ; $t c$, tache caudale ; $e c a$, espace caudal ; pe, pore excréteur ; $c$, cellule excrétrice ; vcc, vestige du corps central ;

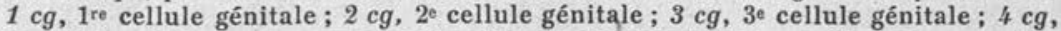
4. cellule génitale ; $p a$, pore anal; $t i$, tache inconstante ; $\propto s$, ébauche œsophagienne; an. $n$. œs., annŁau nerveux œsophagien; ve, ébauche des vaisseaux excréteurs ; st. c., striation cutículaire; $1 \mathrm{eb} . g ., 2 \mathrm{eb} . g ., 3 \mathrm{eb} . g ., 4 \mathrm{eb} . g$. , $1^{\text {re }}, 2^{e}$ e $3^{\text {e }}$ et $4^{\text {e }}$ ébauches génitales ; $x, x^{\prime}, y, y^{\prime}, z, z^{\prime}$, raccords des tronçons des microfilaires A, B et C. 
du trentième jour la mortalité s'accrut rapidement, sans doute par épuisement du milieu, et j'observai les derniers embryons vivants et nettement mobiles le $32^{\circ}$ jour.

Voici (figures 1 et $2, \mathrm{C}$ ) les résultats acquis au $30^{\circ}$ jour et compa-

TABleau CoMparatif No 2

\begin{tabular}{|c|c|c|c|}
\hline $\begin{array}{l}\text { QUELQUES DIMENSIONS } \\
\text { COMPARÉES ÉVALUÉES } \\
\text { EN MICRONS. }\end{array}$ & $\begin{array}{l}\text { MicrofiLAiRE A } \\
\text { 1" JOUR }\end{array}$ & $\begin{array}{c}\text { Microfilame B } \\
20^{\circ} \text { JoUR }\end{array}$ & $\begin{array}{c}\text { MicrofiLAiRe C } \\
\text { 36. JOUR }\end{array}$ \\
\hline $\begin{array}{l}\text { Longueur totale de la } \\
\text { gaine............... } \\
\text { Largeur maxima de la } \\
\text { gaine.............. } \\
\text { Longueur de l'embryon } \\
\text { Largeur maxima de } \\
\text { l'embryon........... }\end{array}$ & $\begin{array}{c}370 \\
9 \\
296 \\
7\end{array}$ & $\begin{array}{c}\text { plus de gaine } \\
\text { ग) } \\
810 \\
8,5\end{array}$ & $\begin{array}{c}\text { plus de gaine } \\
\text { ") } \\
10\end{array}$ \\
\hline \multicolumn{4}{|c|}{ Nombre } \\
\hline $\begin{array}{r}\text { De l'extrémité céphali- } \\
\text { que à la tache en } \mathrm{V} \\
\text { (ou au pore excré- } \\
\text { teur)............... } \\
\text { De la tache en } \mathrm{V} \text { (ou du } \\
\text { pore excréteur) à la } \\
\text { tache caudale (ou au } \\
\text { pore anal)........... } \\
\text { De la tachecaudale (ou } \\
\text { du pore anal) à l'ex- } \\
\text { trémité caudale..... }\end{array}$ & 120 & 156 & $\begin{array}{r}190 \\
42\end{array}$ \\
\hline $\begin{array}{l}\text { Nombre total des cel- } \\
\text { lules somatiques .... }\end{array}$ & 312 & 365 & 397 \\
\hline
\end{tabular}

rés avec les chiffres moyens d'une microfilaire le jour de la prise de sang : la longueur a crû de $283 \mu$ à $340 \mu$, soit un accroissement de $57 \mu$, c'est-à-dire égal à plus d'un cinquième de la longueur primitive ; la largeur a crû de $5,5 \mu$ à $10 \mu$ et même, dans certains cas, à $11 \mu$, soit le double de la largeur primitive. L'accroissement de taille, in vitro, affecte donc surtout la largeur de l'embryon et cela cor- 
respond parfaitement à l'épaississement des embryons de filaire observé par Low dans les muscles du moustique hôte intermédiaire, accroissement en largeur qu'il a dénommé d'une façon imagée " stade saucisse ».

L'espace céphalique, à ce stade, présente à peu près la même grandeur, $6 \mu$; immédiatement après lui, les cellules somatiques se sont multipliées, condensées, pour donner vraisemblablement une ébau-

Tableau comparatif No 3

\begin{tabular}{|c|c|c|c|}
\hline $\begin{array}{l}\text { DistaNCeS DE L'EXTRÉMitÉ } \\
\text { CÉPHALIQUe aUX PRINCIPAUX } \\
\text { ORGANES }\end{array}$ & $\begin{array}{c}\text { Microfilaire A } \\
\text { 1" Jour }\end{array}$ & $\begin{array}{c}\text { MicrofilatRe B } \\
20^{\circ} \text { jotr }\end{array}$ & $\begin{array}{c}\text { Microfilaire C } \\
30^{\circ} \text { jour }\end{array}$ \\
\hline $\begin{array}{l}\text { Espace céphalique.... } \\
\text { Tache oblique (ou an- } \\
\text { neau nerveux œso- } \\
\text { phagien)............. } \\
\text { Tache en V(ou ébauche } \\
\text { excrétrice).......... } \\
\text { Corps central (milieu). } \\
\mathrm{T} \text { a che c a u dale (ou } \\
\text { ébauche génitale).... } \\
\text { Extension de l'ébauche } \\
\text { génitale (pore anal } \\
\text { compris)........... } \\
\text { Tache inconstante..... } \\
\text { Espace caudal (sans cel- } \\
\text { lule somatique)....... }\end{array}$ & $\begin{array}{c}6 \\
\\
58 \\
90 \\
170 \\
237\end{array}$ & $\begin{array}{c}7 \\
56 \\
85 \\
\text { a disparu } \\
240\end{array}$ & $\begin{array}{c}6 \\
\\
55 \\
87 \\
\text { a disparu } \\
184\end{array}$ \\
\hline
\end{tabular}

Ces valeurs numériques sont exprimées en $u$.

che pharyngienne; la tache oblique, anuclée,e, a fait place à une ébauche œsophagienne ou nerveuse, située à la même distance de la tête $(55 \mu)$ et caractérisée par une multiplication cellulaire très nette; à la tache en $\mathrm{V}$ se sont substitués un pore excréteur bien dessiné, une ébauche des cellules et des vaisseaux excréteurs, tous situés $(87 \mu)$ à la distance habituelle qui sépare la tache en $\mathbf{V}$ de l'extrémité céphalique ; la $1^{\text {re }}$ cellule génitale nettement apparue avant le $20^{\circ}$ jour, s'est multipliée pour donner une première ébauche génitale ; la tache caudale qui avait été déjà remplacée par les $2^{\circ}, 3^{\circ}, 4^{\circ}$ cellules génitales et par le pore anal, s'est transformée en $2^{\circ}, 3^{\circ}, 4^{\circ}$ ébau- 
ches génitales, très allongées par multiplication nombreuse aes cellules génitales primitives et en pore anal; la tache inconstante ellemême s'est organisée pour donner une ébauche à laquelle il est bien difficile d'assigner encore une signification; cet accroissement de taille et ces ébauches d'organes sont dus à la multiplication des cellules somatiques et génitales dont le nombre total est passé de 312 à 397 ; à savoir : de l'extrémité céphalique au pore excréteur, le nombre des cellules a crû de 120 à 165 ; il a crû de 164 à 190 dans l'espace compris entre le pore excréteur et le pore anal, et de 28 à 42 entre le poŕe anal et l'extrémité caudale. Les noyaux somatiques sont, dans l'ensemble, plus petits que les noyaux des cellules des embryons non cultivés. En somme, c'est au niveau des ébauches d'organes que se font les divisions nucléaires les plus nombreuses (voir tableau comparatif $n^{\circ} 2$ ) et il est remarquable de constater que l'accroissement en longueur de l'embryon est dû surtout au développement précoce et important des ébauches dites génitales 2,3 et 4 (consulter le tableau comparatif $n^{\circ} 3$ ). Leur extension est passée, en effet, de $5 \mu$ à $85 \mu$.

Notons encore la grande netteté de la striation cuticulaire présentée par ces microfilaires au $30^{\circ}$ jour de culture.

\section{RÉSUMÉ}

$1^{\circ}$ Nous avons pu cultiver, in vitro, pendant 32 jours des microfilaires de Bancroft, en sérum non chauffé, à la température du laboratoire et en employant des techniques bactériologiques simples.

$2^{\circ}$ Les embryons, dans ces conditions de culture, perdent leur gaine en quelques jours. Ce phénomène parait lié à l'augmentation de la viscosité du milieu plutôt qu’à la baisse de sa température.

$3^{\circ}$ Dans ces mêmes conditions expérimentales, le corps central des microfilaires disparaît progressivement et complètement en une vingtaine de jours environ.

$4^{\circ} \mathrm{Au}$ bout de trente jours de culture, les embryons de filaire ont présenté une augmentation notable de leur taille : largeur doublée et accroissement de longueur supérieur au cinquième de la longueur primitive.

$5^{\circ}$ Dans ce même laps de temps, sont apparues nettement diverses ébauches digestives, excrétrices et génitales, formées par la multiplication active des cellules somatiques et génitales des embryons. 


\section{BibLIOGRAPHIE}

Bach (F.-W.). - Ueber die « Microfilarien Kulturen » von Wellman und Johns, nebst Bemerkungen ueber die Messung der Mikrofilarien. Centralbl.f. Bakt. und Parasit. Orig., LXX, 1913, pp. 50-60.

Brumpt (E.). - Précis de Parasitologie. 40 édit., Masson et Cie, Paris, 1927, p. 728.

Coutelen (F.). - Essai de culture in vitro de scolex et d'hydatides échinococciques. Ann. Parasitol., V, 1927, pp. 1-27.

- Sur l'évolution vésiculaire in vitro des scolex échinócocciques. Ann. Parasit., V, 1927, pp. 239-242.

- Contribution à l'étude de la filariose des Grenouilles. Infection et surinfection expérimentales. Ann. Parasitol., V, 1928, pp. 243-244.

- Contribution aux essais de culture in vitro d'embryons de filaires. Bull. soc. path. exot., XXI, 1928, p. 316.

- Essai de culture in vitro du Cénure sérial. Vésiculation des scolex. C. R. Soc. biol., C, 1929, p. 619.

Folex (H.). - Etudes morphologiques sur les microfilaires à gaine (Mf. bancrofti et Mf. diurna). Observations faites chez les tirailleurs sénégalais d'Algérie. Ann. Inst. Past., XXVII, 1913, p. 50.

Füllebonn (F.). - Filariosen des Menschen. Handbuch der pathogenen Mikroorganismen, VI, 1929, p. 1068.

Johns (F. M.) et Quenens (P. L.). - Further note on the growth of filarial embryos in vitro. American Journ. trop. dis. and prev. med., I, 1914, pp. 620-625.

KoRkE (V. T.). - Observations on filariasis in some areas in British India. Indian Journal of medical research, XVI, 1928, p. 187.

Low (G. C ). - The life of filarial embryos outside of the body. Journ. Trop. Med. and Hyg., XV, 1912, pp. 338-339.

Manson-Bahr (Ph.). - Manson's Tropical Diseases. Cassel and Cie, London, 1925, p. 505 .

Nagano (K.). - Beitrag zur « Kultur » der Mikrofilarien ausserhalb des Wirtskörpers. Arch. f. Schiffs u. Trop. Hyg., XXVII, 1923, p. 178-185.

Penel (R.). - Les filaires du sang de l'homme. F.-R. de Rudeval, Paris, 1904.

TакеShiтa (Sh ) et ОкUda (M.). - On the cultivation of Bancroft's filarial larvæ and animal inoculation experiments. Igaku Chuo Zasshi (Central Jl. of med.), XXIII, n 3 . Analyse dans Japan med. World, V, 1925, p. 296 et Trop. dis. Bull., XXIII, 1926, p. 255.

Wellman (C.) et Johns (F. M.). - The artificial culture of filarial embryos. A preliminary note. Journ. Amer. Med. Assoc., LIX, 1912, p. 1531.

Laboratoire de Parasitologie de la Faculté de Médecine de Paris 